\title{
Using Circuit Transformations for the Evaluation of Amplifier Parameters
}

\author{
Franquelim F. Ferreira, FEUP
}

\begin{abstract}
A technique for signal analysis of electronic circuits, using simple circuit transformations, is presented. The circuit transformations are based on the application of the Thévenin's and Norton's theorems. The method is an alternative to directly solving the circuit equations, and is especially useful in some cases for the evaluation of gain and resistance seen from two nodes. A set of examples is presented. Though the procedure is a general method, it is better to regard it as an alternative technique, specially useful in several cases where it can help the student to grasp the operations and get insight into the role played by some configurations used in amplifier circuits.
\end{abstract}

\section{Introduction}

In electronic circuit analysis the evaluation of gain and input/output resistance is an essential step for the understanding of the circuit characteristics and behaviour. The resistance seen from two nodes of a circuit is also used in the time constants method for frequency response analysis of amplifiers.

Nowadays, the students have easy access to simple circuit analysis computer programs. However, in many simple circuits the direct evaluation of parameters is easy to perform: the equivalent circuit is drawn substituting the transistors by their models; the application of the Kirchhoff's laws leads to a set of equations from which the parameters can be evaluated.

Nevertheless, this methodological simplicity does not seem to help the students to develop enough sensitivity to the circuit behaviour.

In our opinion, a good performance of the students in the simplified analysis is a fundamental tool to get a high degree of competence in circuit synthesis and design. For this purpose we have been encouraging our students to solve this type of problems using simple circuit transformations, based on the application of the Thévenin's and Norton's theorems, which we believe is an efficient way to give insight to the circuit behaviour.

Thévenin's and Norton's theorems are essential tools in amplifier circuit analysis. Any textbook of Electronics, like [1] and [2], refer to them and recommend their application. Even today, studies and work on their application are published, [3], [4].

The circuit transformations, proposed in this paper, simply exploit these topics further.

Beyond other advantages, the mathematical operations to be carried out using this technique are frequently so simple that dispense the calculator, allowing the student, in each step, to get a tight control of what he/she is doing - in opposition to the use of CAD methods or even a mechanical equation solving procedure.

This paper intends to demonstrate a circuit transformation technique, that is simply an alternative to the classic method consisting in solving the circuit equations. For this purpose, we present a set of examples through which we believe the method becomes clear. 


\section{Example 1}

Consider the common source enhancement MOSFET amplifier with depletion load of fig. 1.

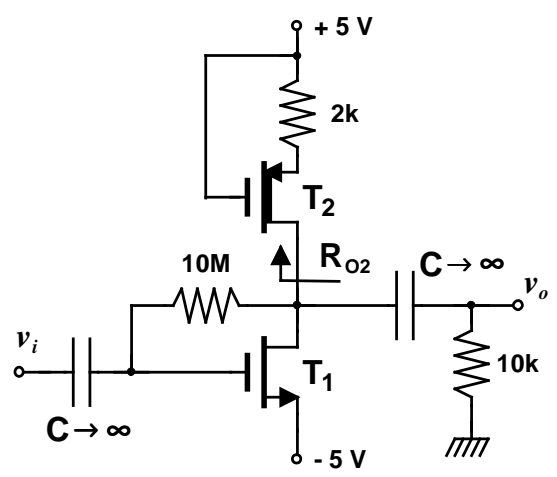

Fig. 1

We want to obtain the drain load resistance of $T_{1}$, which is equal to the output resistance of $T_{2}$, looking into the drain, $R_{o 2}$.

The DC analysis allows us to conclude that the small-signal model parameters of $\mathrm{T}_{2}$ are:

$$
g_{m 2}=1 \mathrm{~mA} / \mathrm{V} \quad r_{o 2}=50 \mathrm{k} \Omega \quad \mu_{2}=g_{m 2} r_{o 2}=50
$$

Fig. 2(a) shows the equivalent circuit to be used for the evaluation of $R_{o 2}$, that can be redrawn as in fig. 2(b).

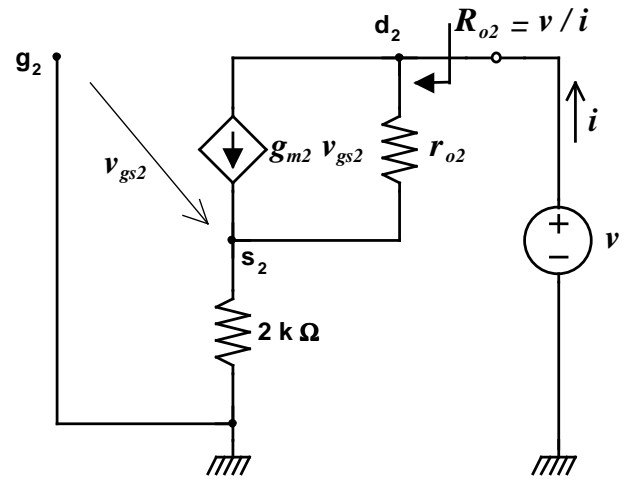

(a)

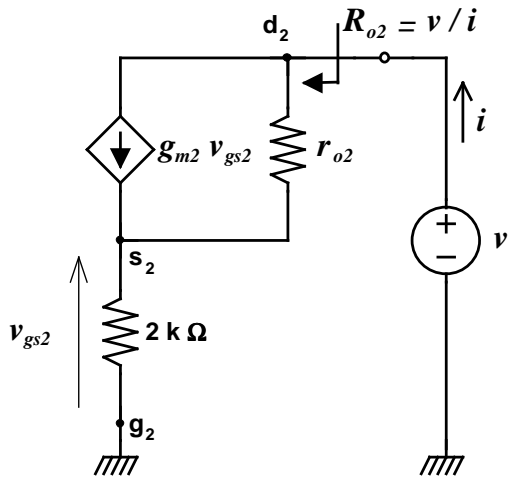

(b)

Fig. 2

Substituting the Norton configuration $\left\{\begin{array}{lll}g_{m 2} & v_{g s 2}, & r_{o 2}\end{array}\right\}$ by its Thévenin's equivalent, results the circuit of fig. 3(a).

Since

$$
v_{g s 2}=-i \times 2 \mathrm{k} \quad \text { and hence } \quad \mu_{2} v_{g s 2}=-i \times 100 \mathrm{k}
$$

we obtain the circuit of fig. $3(\mathrm{~b})$. 


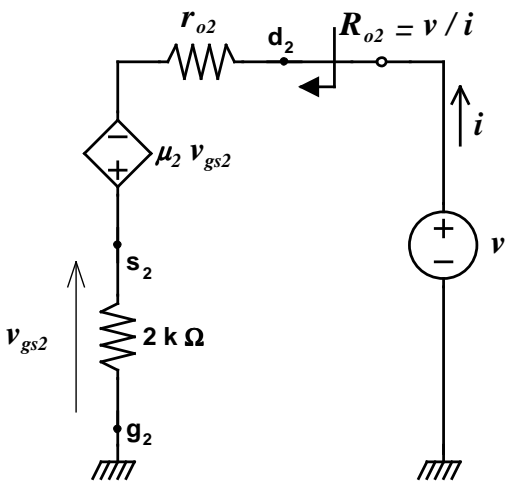

(a)

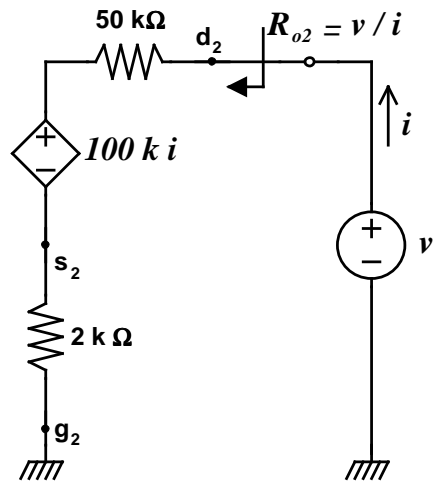

(b)

Fig. 3

Finally, applying the voltage source absorption theorem to the controlled source $100 \mathrm{k} . i$, and since its current is its own controlling current can be replaced by a $100 \mathrm{k} \Omega$ resistor.

The resulting circuit (fig. 4) shows, by simple inspection, that the desired resistance $R_{o 2}$ is

$$
R_{o 2}=50 \mathrm{k}+100 \mathrm{k}+2 \mathrm{k}=152 \mathrm{k} \Omega
$$

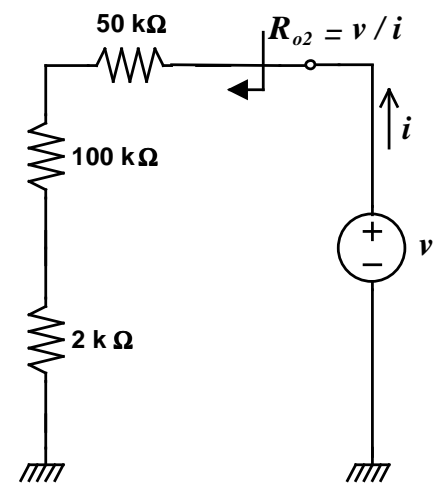

Fig. 4

The effect of transistor $\mathrm{T}_{2}$ in increasing the resistance $R_{o 2}$ is provided by the current source $g_{m 2}$ $v_{g s 2}$, which can be easily seen, given its substitution by the $100 \mathrm{k} \Omega$ resistor, in series.

\section{Example 2}

The evaluation of input and output resistance can be seen as a particular case of the more general problem of a resistance seen from two nodes.

The procedure to follow is well known: reduce all independent sources to zero and apply a test voltage source $v$ between the two nodes; the wanted resistance is equal to $v / i$, being $i$ the current provided by the test source.

Accordingly, evaluating the resistance seen by the intrinsic capacitances of transistors for the application of the time constants method or for the computation of the time constants associated to the dominant pole can be done following the very same procedure.

As an example, we consider the problem of evaluating the resistance seen by $C_{\pi l}\left(R_{\pi l}\right)$, assuming $C_{\mu l}$ as an open circuit [2], [5] (fig. 5). 


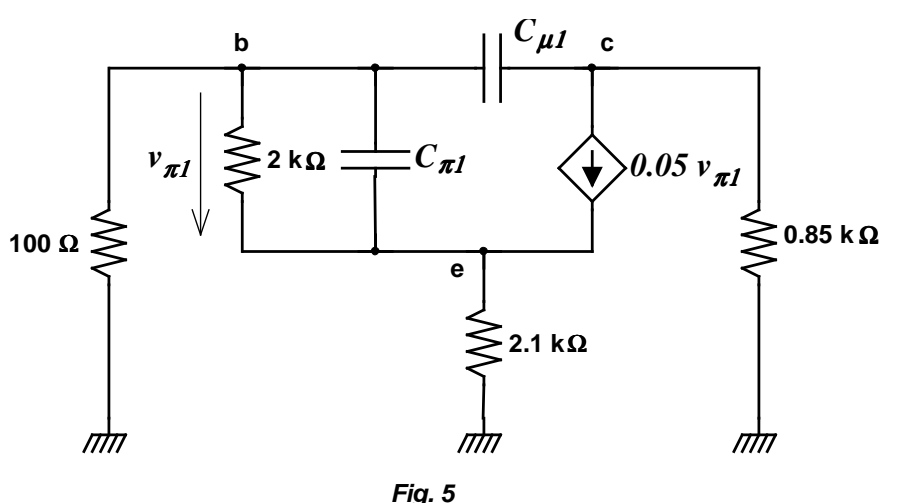

By direct inspection of the circuit we conclude that $R_{\pi l}=2 \mathrm{k} \Omega / / R$, being $R$ the resistance shown in the fig. 6(a). Voltage $v_{\pi l}$ coincides with the test voltage $v$ to be applied for evaluating $R$. In fig. 6(b), $v_{\pi l}$ was substituted by $v$.

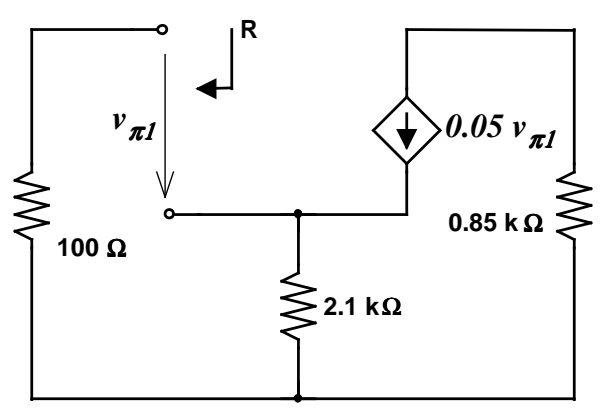

(a)

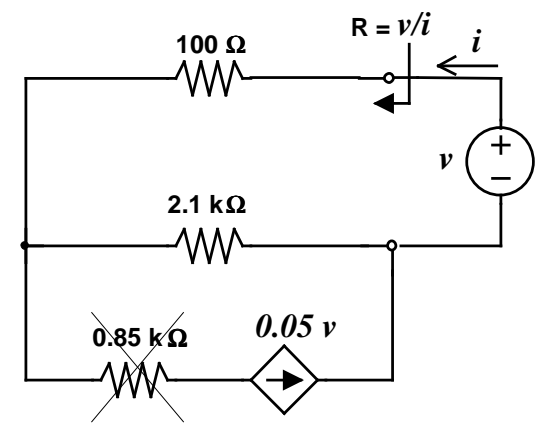

(b)

Fig. 6

This circuit suggests that the application of the Thévenin's theorem to the pair constituted by the current source and the $2.1 \mathrm{k} \Omega$ resistor can be useful. The $0.85 \mathrm{k} \Omega$ resistor does not affect the result.

The circuit shown in the fig. 7(a) is thus obtained and can be redrawn as it is illustrated in fig. 7(b):

$$
i=\frac{106 v}{2,2 \mathrm{k}} \quad \text { and hence } \quad R=\frac{v}{i}=\frac{2,2 k}{106} \cong 21 \Omega
$$

giving rise to

$$
R_{\pi 1}=2 \mathrm{k} / / 21 \cong 21 \Omega
$$

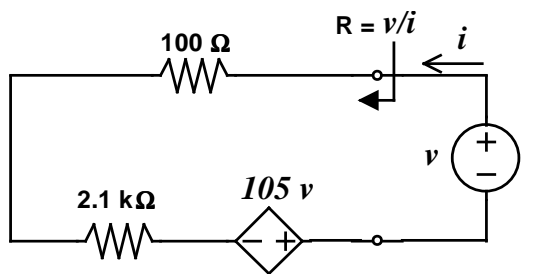

(a)

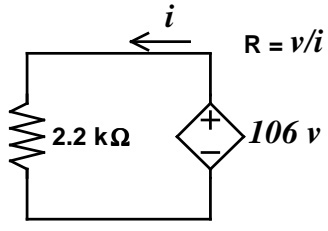

(b)

Fig. 7

Once more the role of the controlled voltage source becomes obvious, this time in decreasing the total value of $R$. 


\section{Example 3}

Let us now use this technique to evaluate the input resistance and gain of the circuit in fig. 8(a). The advantage of this procedure turns evident if, e.g., the emitter resistance is small and the collector resistance very high, in which case the common aproximations $R_{i} \cong r_{\pi}+\left(1+\beta_{o}\right) R_{E}$ and $A_{v} \cong-R_{C} / R_{E}$ are not valid.

So, let us consider the low frequency voltage gain and input resistance of an intermediate stage (fig. 8(a)), using the equivalent circuit of fig. 8(b) and assuming that the transistor has the following model parameters: $r_{\pi}=25 \mathrm{k} \Omega, \beta_{o}=200$ e $r_{o}=0.5 \mathrm{M} \Omega$.

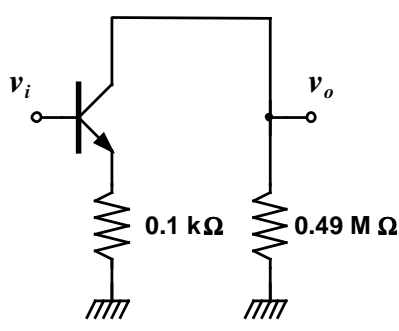

(a)

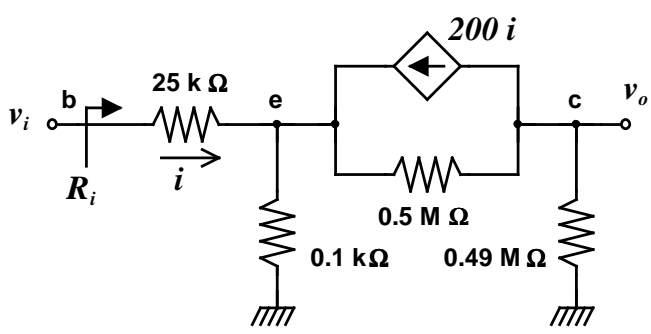

(b)

Fig. 8

Applying the Thévenin's theorem to the circuit, to the right of the emitter node, we obtain the circuit of fig. 9(a), and then, including the $0.1 \mathrm{k} \Omega$ resistance, and applying Thévenin's theorem again, we come to the circuit of fig. 9(b), where we should notice that the values $10^{4}$ in the source, and $0.1 \mathrm{k} \Omega$ are very accurate approximations.

Finally, using the voltage source absorption theorem, we obtain the circuit of fig. 9(c), where it is obvious to conclude that $R_{i} \cong 35 \mathrm{k} \Omega$.

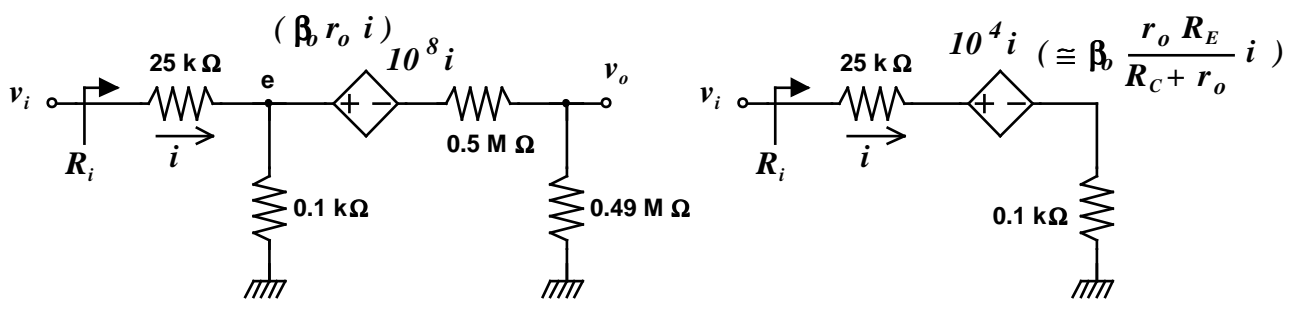

(a)

(b)

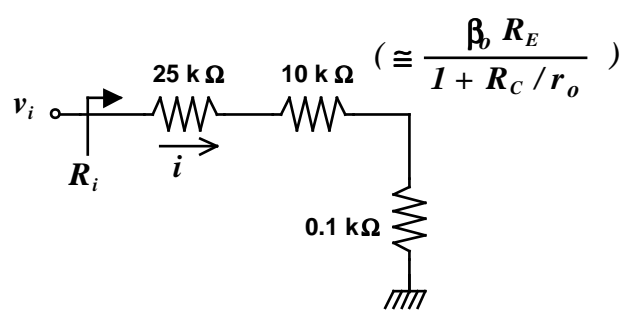

(c)

Fig. 9

We should notice, once more, that the procedure favours the understanding of the role played by the source $\beta_{o}\left(r_{o} R_{C} /\left(R_{C}+r_{o}\right)\right) i$ (corresponding in fig. 9 (b) to the source $\left.10^{4} i\right)$ in increasing the input resistance, $R_{i}$ in a way that depends on the ratio $R_{C} / r_{o}$.

Moving now to the evaluation of the voltage gain, let us come back to the circuit of fig. 9(a).

Since $i=v_{i} / R_{i}$, the source $10^{8} i$ can be re-written as a function of $v_{i}$

$$
10^{8} i=\frac{10^{8}}{35 \mathrm{k}} v_{i}=\frac{10^{5}}{35} v_{i}
$$


Applying the Thévenin's theorem to the left of the point labeled $\mathbf{e}$, we obtain the circuit of fig. 10 (a), where the values 0.004 , in the source, and $0.1 \mathrm{k} \Omega$ are very accurate approximations, and respectively much smaller than the controlled voltage source and the series of the other two resistors.

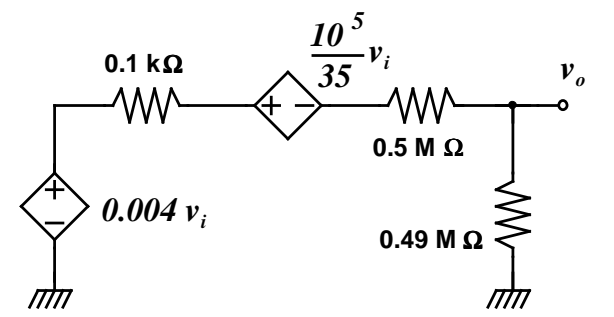

(a)

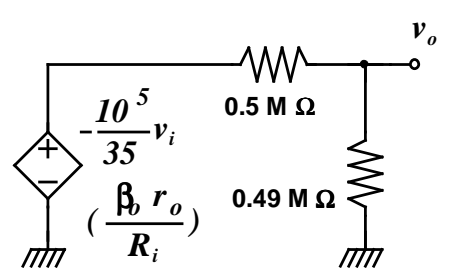

(b)

Fig. 10

The gain can then be given by:

$$
\frac{v_{o}}{v_{i}} \cong \frac{1}{2}\left(-\frac{10^{5}}{35}\right) \cong-1400
$$

which is smaller than $R_{C} / R_{E}$, because the controlled source is $\beta_{o} r_{o} / R_{i}$ and it is divided between $r_{o}$ and $R_{C}$.

\section{Conclusion}

The circuit transformation techniques, described and demonstrated in this paper, constitute an alternative to the general method of writing and solving the circuit equations. However, this alternative does not provide, in all cases, the amount of simplifications shown in the illustrating examples. Its merit comes from the fact that it helps making clear the role of some of the circuit components.

On the other hand, the practice of these techniques in the initial stages of learning will help the student to get insight and better grasp of the operations to be done in the circuits analysis. Although the application of these techniques is not error free, it is less prone to mistakes since it allows the student to keep a tight control of the magnitude of the circuit parameters, along the whole process.

\section{Acknowledgement}

The author wishes to thank Prof. Guedes de Oliveira, who has encouraged him to write this paper and has offered him valuable suggestions about its content.

\section{References}

[1] J. Millman and A. Grabel, Microelectronics, 2nd. ed. New York: McGraw Hill, 1987.

[2] A. Sedra and K. Smith, Microelectronic Circuits, 3rd. ed. New York: Saunders HBJ, 1991.

[3] Mohamed F. Moad, “On Thévenin's and Norton's Equivalent Circuits"., IEEE Tran Educ., vol. E-25, no. 3, pp. 99-102, August 1982.

[4] Lin-Ming Jin and Shu-Park Chan, "A Unified and Efficient Approach for Determining Thévenin (Norton) Equivalent Circuits.", IEEE Tran Educ., vol. 32, no. 3, pp. 408-410, August 1989.

[5] P. E. Gray and C. L. Searle, Electronic Principles, New York: Wiley, 1969. 